\title{
Effect of Testosterone on Memory and BDNF Levels of Hippocampus in Gonadectomized Diabetic Rats
}

\author{
Mahboubeh Ebrahimzadeh ${ }^{1}$, Parviz Shahabi ${ }^{*}$, Gisou Mohaddes ${ }^{2}$, \\ Shirin Babri ${ }^{2}$, Mostafa Mohammadi ${ }^{3}$, Alireza Moslem and \\ Mohammad Mohammad-zadeh ${ }^{4 *}$
}

\begin{abstract}
${ }^{1}$ Tabriz University of Medical Sciences, International Branch Aras, Tabriz- Iran ${ }^{2}$ Neuroscience Research Center, Tabriz University of Medical Sciences, Tabriz-Iran ${ }^{3}$ Department of Physiology, Tabriz University of Medical Sciences, Tabriz-Iran ${ }^{4}$ Cellular and Molecular Research Center, Sabzevar University of Medical Sciences, Sabzevar- Iran.
\end{abstract}

DOI: http://dx.doi.org/10.13005/bbra/1921

(Received: 30 September 2015; accepted: 07 November 2015)

The prevalence of cognitive impairment in diabetic patients is more than in non-diabetic ones. BDNF and sex hormones levels play important roles in learning and memory. In this study, the effect of testosterone on BDNF expression and memory was investigated in 60 male Wistar rats were randomly divided into 6 groups $(n=10)$ : control (C), Diabetic (Dia), Diabetic-Sham (Sh), Diabetic-Gonadectomized (Dia-Gdx), DiabeticTestosterone (Dia-T), and Diabetic-Gonadectomized-Testosterone (Dia-Gdx-T). Diabetes was induced by STZ $(60 \mathrm{mg} / \mathrm{Kg})$. Testosterone $(2 \mathrm{mg} / \mathrm{kg} / \mathrm{day})$ was administered intraperitoneally for 6 weeks. The BDNF levels in the hippocampus were assessed by ELISA kit. Novel object recognition (NOR) test was performed for the investigation of recognition memory. Induction of diabetes did not significantly change the BDNF levels of hippocampus, where as Gonadectomy or testosterone administration in diabetic animal's significantly reduced BDNF levels. Short-term memory significantly was reduced in Dia-Gdx, Dia-T, and Dia-Gdx-T groups compared to the control group; however, Gonadectomy or testosterone therapy had no significant effects on it in diabetic rats. BDNF levels and short-term memory decreased in diabetic groups that received or were depleted from testosterone. It is possible that decrease of BDNF gene expression in the hippocampus caused a short-term memory disturbance.

Key words: Learning and memory, Diabetes, BDNF, Testosterone

Diabetes mellitus (DM) is a common metabolic disease of endocrine system and is caused by lack of cellular uptake of blood glucose by reducing insulin (T1DM) ${ }^{1}$ or the body cells resistance to the insulin (T2DM $)^{2}(1)$. In many cases, diabetes leads to a reduction in neural activity and an increase in risk factors of a variety of neurological diseases, such as stroke ${ }^{2}$. Slow progressive damages in brain tissue are created

\footnotetext{
* To whom all correspondence should be addressed. There is two corresponding authors Tel/fax: 098- 051-44264462

E-mail: mohamadzadehm@medsab.ac.ir Tel/Fax:098-41-33364664; E-mail: parvizshahabi@gmail.com
}

by diabetes. In recent years, a moderate or severe decrease in cognitive functions have been reported in people with diabetes type 1 and $2^{2,3}$. The prevalence of cognitive impairment in diabetics is more than in non-diabetics ${ }^{4}$. Cognitive functions including a number of complex behaviors such as problem solving, attention, planning are mediated by frontal lobe (executive function) ${ }^{5}$.

During diabetes, the brain is no longer able to provide glucose required for many processes such as memory. This effect is especially evident in the hippocampus, which is involved in memory and learning ${ }^{6}$. Hyperglycemia, which is a marker of diabetes, can lead to a decrease in cognitive memory by different mechanisms. Acute 
changes in glucose concentration can alter the brain regional blood flow; on the other hand, they are able to induce osmotic changes in brain neurons ${ }^{7}$. The rate of blood glucose enhancement is directly related to oxidative stress. Considering that the antioxidant protection system of the brain is not powerful, this process can harm the brain cells progressively, particularly in hippocampus ${ }^{8}$.

Sex hormones such as androgens regulate the proliferation, differentiation and homeostasis of the cells. The role of androgens has been confirmed in memory, learning and cognitive memory. The functional mechanism of sex hormones have genomic and non-genomic effects on peripheral target organs ${ }^{9}$. Based on studies, a direct link exists between the amount of free testosterone in blood and cognitive memory. According to studies, $13 \%$ of people older than 60 years old and $32 \%$ older than 85 years old with low levels of blood free testosterone suffer from cognitive memory disorders ${ }^{10}$. On the other hand, the amount of free testosterone in the blood of diabetic patients is lower than in healthy people ${ }^{11}$. $\mathrm{BDNF}^{3}$ is a $27-\mathrm{kDa}$ polypeptide member of the neurotrophins family. This protein plays an important role in the survival, growth, maintenance of neurons, learning and memory ${ }^{12,13}$. Also, BDNF factor has been shown to express in original cells of hippocampus and play a significant role in activity-dependent synaptic plasticity ${ }^{14,15}$. BDNF prevents apoptosis by stimulating the synthesis of anti-apoptotic factors like BCL-2 ${ }^{16}$. It is reported that some diseases such as Alzheimer's and depression are associated with reduction in BDNF levels ${ }^{17}$. On the other hand, studies have shown that the levels of BDNF factor are reduced during hyperglycemia in T1DM patients ${ }^{18}$.

Therefore, while considering the effects of diabetes and testosterone on cognition and memory in this study, we evaluated the effect of testosterone on the expression of BDNF gene and cognitive memory in diabetic animals.

\section{MATERIALSAND METHODS}

\section{Animals}

In this study, 60 male Wistar rats (250-350 g and, 6-7 months old and obtained from animal house of Tabriz University of Medical Science) were used. They were randomly divided into 6 groups of 10, including control (C), Diabetic (D), Diabetic-Sham (Sh), Diabetic-Gdx ${ }^{44}$ Gonadectomized (Dia-Gdx), Diabetic-Testosterone (Dia-T), and Diabetic-Gdx-Testosterone (Dia-Gdx$\mathrm{T})$.Animals were kept in a temperature-controlled room $\left(22 \pm 3^{\circ} \mathrm{C}\right)$ with $12 \mathrm{~h}$ light $/ 12 \mathrm{~h}$ dark cycle (lights on at 6:00 a.m.). Food and water was provided ad libitum. Experimental procedures were in accordance with the Regional Ethics Committee of Tabriz University of Medical Science ${ }^{19}$.

\section{Surgical procedures for gonadectomy}

Animals were anesthetized using intraperitoneal injection of Sodium pentobarbital (50 mg/kg). Then, the rats were placed in supine position and a horizontal incision was performed in scrotum and the testes were tied off and removed bilaterally with a cut distal to the ligature. After the surgery, the incision was closed using suture. Gonadectomy was performed one week prior toinduction of diabetes. In the Diabetic-Sham group, an incision was made in the scrotum but testes were not removed.

\section{Drugs}

Subcutaneous injection of testosterone propionate (UNIGEN Life Sciences), $2 \mathrm{mg} / \mathrm{kg} /$ day, for 6 days beginning at castration, was performed for 6 weeks ${ }^{20}$ in Dia-T and Dia-Gdx-T. Testosterone propionatewas dissolved in $\mathrm{DMSO}^{521}$.

\section{The induction of diabetes}

In order for induction of diabetes to occur, a single dose $(60 \mathrm{mg} / \mathrm{kg}$ ) of Streptozotocin (STZ) prepared by $0.1 \mathrm{M}$ of citrate buffer $(\mathrm{pH}=4.5)$ was administered intraperitoneally (i.p.) ${ }^{19}$. After $72 \mathrm{~h}$, a small injury was created in the tail of animals and blood samples were obtained from tail vein. Blood glucose levels were measured using glucometer (Arkray, Kyoto, Japan) and the levels higher than $300 \mathrm{mg} / \mathrm{dl}$ were considered as an index of diabetes.

\section{Measurement of BDNF protein}

At the end of the experiment, animals were anesthetized with sodium pentobarbital (50 mg/ $\mathrm{kg}$ ). The brain was removed and the hippocampus was separated immediately. The tissue was washed with cold 9\% normal saline and frozen in liquid nitrogen. During the homogenization, the hippocampus tissue was homogenized by glass homogenizer in a special buffer on ice and was kept for further tests. BDNF protein was assessed using the BDNF E-Max ELISA kit (Promega, USA) according to the manufacturer's instructions. 


\section{Novel objects recognition (NOR) test}

The Novel Object Recognition (NOR) test is used to evaluate cognitive function, particularly recognition memory, in rodents. This test is based on the spontaneous tendency of rodents to spend more time exploring a novel object than a familiar one $^{22}$. Use of this test was for reasons including its non-invasive nature, there being no need for a stimulus, no need to reward or punish the animal and the animal not needing to spend a long time for habituation to the test ${ }^{23}$. Interestingly, the results of these tests are completely influenced by hippocampus disorders ${ }^{24}$.

The experiments were performed using an open field box in size of $50 \mathrm{~cm} \times 25 \mathrm{~cm} \times 50 \mathrm{~cm}$ and a video recording system (Logitech pro 9000). Behavioral analysis was performed by video check. This protocol was repeated for $10 \mathrm{~s}, 60 \mathrm{~s}, 10 \mathrm{~min}$ and $90 \mathrm{~min}$ for the investigation of short-term memory. On the third day (after $24 \mathrm{~h}$ ), the rats were placed in the test box and they touched the objects for 3 minutes. The test was performed on the fourth day (48 h) as on the third, in order to evaluation long-term memory (defined as the test session). A ratio of the amount of time spent exploring each object (at training session) or substitution with novel one (at test session) upon the total time spent analyzing both objects, were selected as Recognition Index (RI) for investigation of memory preference ${ }^{26}$. The RI was calculated by dividing the novel object exploration time by the total exploration time (novel/novel+ familiar investigation $)^{25}$. The test box and objects were cleaned by $70 \%$ of ethanol after each use ${ }^{26}$.

\section{Body weight measurement}

Body weights were measured before and after induction of diabetes. Then, body weight percent was calculated by the following formula:
$\mathrm{BW} \%=(\mathrm{WB} " \mathrm{WA}) / \mathrm{WB} \times 100$, where $\mathrm{WB}$ is the weight before induction of the diabetes and WA indicates the weight after applying the interventions.

\section{Data analysis and statistics}

For statistical analysis, SPSS16 software was used. Data were analyzed using One-Way ANOVA. Then, Fisher's Least Significant Difference (LSD) post hoc test was performed and $P$ values less than 0.05 were considered significant. The data were expressed as the Mean \pm SEM.

\section{RESULTS}

\section{The result of BDNF gene expression in hippocampus}

The results of our study indicated that induction of DM by STZ did not significantly $(\mathrm{P}>0.05)$ change BDNF gene expression in the hippocampus of the diabetic group compared with the control group. Castration in diabetic animals led to a significant $(\mathrm{P}<0.05)$ decrease in $\mathrm{BDNF}$ gene expression in Dia-Gdx group compared with C, Dia and sh groups. Testosterone treatment for 6 weeks in diabetic animals caused a significant $(\mathrm{P}<0.05)$ decrease in BDNF gene expression compared with C, Dia, sh, and Dia-Gdx-T groups, while BDNF gene expression in Dia-Gdx-T group returned back to normal levels (Figure 1).

\section{Novel objects recognition (NOR) test}

In this test, recognition index (RI) was used as a measuring scale. The amount of RI demonstrate memory preference, with diminution in RI indicating significant decrease in short-term memory ( $10 \mathrm{~s}, 60 \mathrm{~s}, 10 \mathrm{~min}$ and $90 \mathrm{~min}$ ) or long-term memory ( $24 \mathrm{~h}$ and $48 \mathrm{~h}$ ). Castration in diabetic rats led to a reduction in recognition index, and this amount was significant $(\mathrm{P}<0.05)$ in short-term

Table 1. Comparison of the weight changes percentage in rats (\%) in different groups of rats, 6 weeks after the induction of diabetes and Testosterone treatment. (One-way ANOVA test)

\begin{tabular}{lcc}
\hline Groups of rats & The number Rats (n) & Change in body weight (\%) \\
\hline Control & 10 & 0.8 \\
Diabetic & 10 & $-11.5^{*}$ \\
Diabetic-Sham & 10 & $-7.86^{*}$ \\
Diabetic-Gdx & 10 & $-12.21^{*}$ \\
Diabetic-Testosterone & 10 & $-7.69^{*}$ \\
Diabetic-Gdx-Testosterone & 10 & $-13.74^{*}$ \\
\hline
\end{tabular}


memory (10 s and $10 \mathrm{~min})$. A poor increase of RI was seen in $\mathrm{Gdx}$-T rats in comparison to $\mathrm{Gdx}$ group; the amount of this increase was more prominent in long-term memory ( $24 \mathrm{~h}$ and $48 \mathrm{~h}$ ). Statistical analysis also showed that testosterone treatment in diabetic rats significantly decreased RI in shortterm memory (10 s, 1 min and $10 \mathrm{~min}$ ) (Figure 2).

\section{Change in body weight}

Changes in body weight of rats in Dia, Sh, Dia-Gdx, Dia-T and Dia-Gdx-T showed significant decreases in comparison to control group $(\mathrm{p}<0.05)$ and the body weight percentage had no significant increase after testosterone treatment in comparison to Dia-Gdx group ( $\mathrm{p}>0.05$ ) (Table 1).

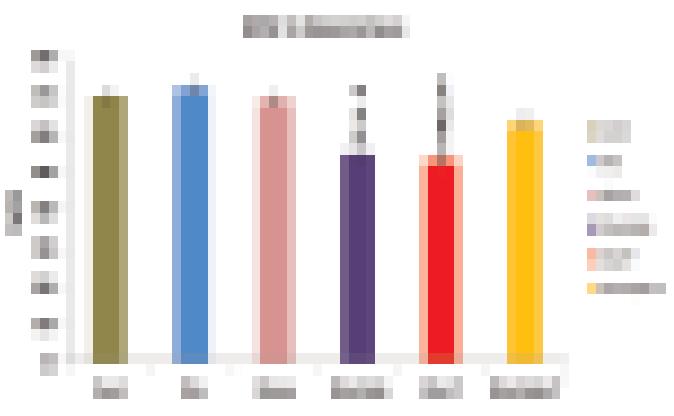

Fig. 1. Comparison of BDNF protein expression of hippocampus tissue into six groups (C, Dia,Sh, DiaGdx, Dia-T,Dia-Gdx-T). Data are shown as mean \pm SEMand $\mathrm{P}<0.05$ was considered significant. (* Indicates $\mathrm{p}<0.05$ in comparison with Dia group. \# indicates $\mathrm{p}<0.05$ in comparison tosh group . $\psi$ indicates $\mathrm{p}<0.05$ in comparison to the $\mathrm{C}$ group. \$ Indicate $\mathrm{p}<0.05$ in comparison toDia-Gdx-T group)

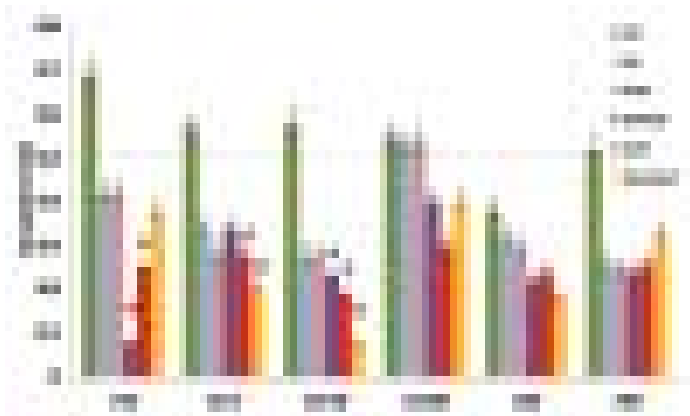

Fig. 2. Comparison of short-term and long-term memory by using RI in novel object recognition test into six groups (C,Dia,Sh, Dia-Gdx,Dia-T, Dia-Gdx-T). Data are shown as mean \pm SEM. $\psi$ Indicates $\mathrm{p}<0.05$ in comparison to the control group.

\section{DISCUSSION}

In this study, NOR test determined that depletion or treatment with testosterone for six weeks in diabetic animals resulted in a decrease in cognitive function and short-term memory. We have also indicated that depletion or treatment with testosterone for six weeks decreases the expression of the BDNF gene in the hippocampus of the diabetic animals.

There is a relationship between cognitive dysfunction and type 1 diabetes. Results of a metaanalysis in 2005 showed that in patients with type 1 diabetes, cognitive dysfunction is characterized by a slowing of mental speed and a diminished mental flexibility ${ }^{27}$. Streptozotocin is used for experimental diabetes mellitus ${ }^{28}$. It can create memory and learning-related disorders in male Wistar rats ${ }^{29}$.

Diabetes is associated with gonadal hormone deficiency. Testosterone deficiency is common in men with diabetes, regardless of the type $^{30}$.This makes the situation more complicated. The relationship between sex hormones and cognitive performance is complicated and unclear. On the one hand, some studies have shown that testosterone depletion or administration does not affect learning and memory ${ }^{31}$; on the other hand, several studies have indicated that androgens impaired $^{32,33}$ or enhanced spatial learning and memory ${ }^{34,35}$. Hawley, in 2013, showed that cognitive memory in castrated rats had no significant difference compared with the rats receiving testosterone after the surgery ${ }^{36}$. Recent studies in adult male rats have found modulatory roles for sex steroids on cognitive and functional memory and other types of cognitive tasks that are sensitive to neural disorders ${ }^{37}$.

According to another study using NOR test, more exploration was observed in detecting new and familiar objects in control and Gdx-T groups compared with Gdx and Gdx-estradiol groups, but long-term memory had a significant decrease after a 1.5 hour delay in Gdx rats which was compensated by injection of testosterone (Not estradiol) ${ }^{38}$. This finding is in line with our findings that long-term memory was not affected in castrated or testosterone treated diabetic animals. Frye and Lacey in 2001, suggested that administering estradiol, progesterone and even 
dihydrotestosterone and other non-aromatizable testosterone positively affects the exploration and discrimination of novel objects in females. Progesterone inhibits learning and memory. Spatial memory is increased by testosterone in rats and this increase is due to estradiol aromatization ${ }^{39}$. The discrepancy between this study and our study is possibly because of the gender difference.

Animal studies suggest that testosterone's role in diagnosis and learning is very important. Reduced androgen results in significant decreases in synaptic density in the hippocampus of rodents and non-human primates. It seems that testosterone has a major role in the survival of neural cells, so testosterone probably is a neuronal protecting factor to repair nerve damage in some neurodegenerative diseases such as diabetes and Alzheimer's ${ }^{40}$. Spritzer at a controversial study demonstrates that gonadectomy had no significant effect on hippocampal cell proliferation but resulted in a reduction of hippocampal cell survival in adult male rats. Androgens increase neuron survival but not cell proliferation within the dentate gyrus ${ }^{41-43}$. Furthermore, in vitro studies have indicated that low doses of testosterone can induce proliferation of neural cells, whereas high doses induce apoptosis in neurons ${ }^{44}$. Thus, the effects of testosterone on hippocampal neurogenesis may be dose-dependent. Low levels of testosterone increase neurogenesis and the amount of BDNF, and high doses of this androgen suppress neurogenesis. Androgens may also influence adult neurogenesis by altering levels of neurotrophic factors within the brain. For example, lower doses of testosterone increase the levels of brain-derived neurotrophic factor (BDNF), and vascular endothelial growth factor (VEGF). Both BDNF and VEGF have been associated with increased survival of newly proliferated neurons within the dentate gyrus $^{45,46}$. Our findings in this study showed that depletion or administration of testosterone in diabetic animals impairs cognitive function and short-term memory.

BDNF, as one of the neurotrophic factors in the brain, plays an important role in some processes such as learning, mobility, behavior, memory and a wide range of responses to oxidative stress $^{47}$. BDNF presumably has a significant role in preservation and development of the nervous system, including regulation of neurogenesis, neuroplasticity and cell survival ${ }^{48}$. Villani, in 2007 , showed that the content and expression of the BDNF gene increases in diabetes mellitus in order to exert a protective effect against the toxic and destructive effects of the disease ${ }^{49}$. Another study demonstrated that BDNF causes the strengthening of the ability of the neurons to reduce ischemic damage and apoptosis in hippocampal cells of diabetic rats ${ }^{50}$. Some research states that reduction of protein BDNF is the primary reason of damage to the central nervous system in patients with diabetes. This disagreement may be due to differences in maintenance duration of diabetic animals following induction of diabetes, as Nitta reported that a reduction in protein BDNF occurs after a 4-week maintenance of diabetic animals following induction of diabetes ${ }^{51}$. In the present study, induction of diabetes with STZ did not change BDNF content of hippocampus.

We also showed that the expression of BDNF was significantly reduced in Gdx and testosterone treated diabetic rats in comparison to the diabetic group. We also observed that injection of testosterone in Gdx rats returned the expression of BDNF levels back to normal values. Parallel with our study, the results of recent studies have shown that gonadectomy in male rats resulted in a significant reduction in the expression of BDNF and PSD-95 in CA1 area. The reduction in expression of BDNF was compensated for by the injection of testosterone in $\mathrm{Gdx}$ rats and increased the expression of $\mathrm{BDNF}^{20}$. several studies also suggest that gonadectomy in male rats leads to a reduction in BDNF and its receptor expression levels in motor neurons of the spinal cord, and the decline was prevented by testosterone replacement ${ }^{49}$. In 1999, Rasika showed that the levels of BDNF were increased after injection of testosterone and lead to neurogenesis stimulation and survival of neurons ${ }^{52}$. The increase in neurological symptoms is improved by the injection of testosterone, possibly due to the inhibition of apoptosis and increases in cell survival by increasing levels of BDNF and the antioxidant defense $^{53}$. This finding is in contradiction with our results that administration of testosterone in diabetic animals decreased testosterone levels of hippocampus and is possibly due to the effects of diabetes on BDND levels of hippocampus.

In conclusion, the results of our study 
showed that the less or more testosterone in diabetic animals decreases expression of BDNF protein, cognitive function, and short-term memory, whereas testosterone depletion or treatment in diabetic animals had no effect on long-term memory.

\section{ACKNOWLEDGMENTS}

This research was financially supported by Cellular and Molecular research Center of Sabzevar University of Medical Sciences. The article is derived from the MSc dissertation of Ms Mahboubeh Ebrahimzadeh entitled "Effect of testosterone on memory and BDNF levels of hippocampus in gonadectomized diabetic rats”.

\section{REFERENCES}

1. Singh, P., Jain, A., Kaur, G. Impact of hypoglycemia and diabetes on CNS: correlation of mitochondrial oxidative stress with DNA damage. Mol Cell Biochem. 2004;260(1):153-9.

2. Biessels, G., Kappelle, L. Increased risk of Alzheimer's disease in Type II diabetes: insulin resistance of the brain or insulin-induced amyloid pathology? Biochem Soc Trans. 2005;33(Pt 5):1041-4.

3. van den Berg, E., Dekker, J.M., Nijpels, G., Kessels, R.P., Kappelle, L.J., de Haan, E.H., et al. Cognitive functioning in elderly persons with type 2 diabetes and metabolic syndrome: the Hoorn study. Dement Geriatr Cogn Disord. 2008;26(3):261.

4. Berchtold, N., Chinn, G., Chou, M., Kesslak, J., Cotman, C. Exercise primes a molecular memory for brain-derived neurotrophic factor protein induction in the rat hippocampus. Neuroscience. 2005;133(3):853-61.

5. Munshi, M., Grande, L., Hayes, M., Ayres, D., Suhl, E., Capelson, R., et al. Cognitive dysfunction is associated with poor diabetes control in older adults. Diabetes care. 2006; 29(8):1794-9.

6. Mendelsohn, A.B., Belle, S.H., Stoehr, G.P., Ganguli, M. Use of Antioxidant Supplements and Its Association with Cognitive Function in a Rural Elderly Cohort The MoVIES Project. Am J Epidemiol. 1998;148(1):38-44.

7. Vijayakumar, T., Sirisha, G., Farzana Begam, M., Dhanaraju, M. Mechanism linking cognitive impairment and diabetes mellitus. Eur j Appl sci. 2012; 4(1): 01-5.
8. Peacock, J.M., Folsom, A.R., Knopman, D.S., Mosley, T.H., Goff, D.C., Szklo, M. Dietary antioxidant intake and cognitive performance in middle-aged adults. Public health nutrition. 2000; 3(3): 337-43.

9. Palumbo, M., Salvestroni, C., Gallo, R., Guo, A-L., Genazzani, A., Artini, P., et al. Allopregnanolone concentration in hippocampus of prepubertal rats and female rats throughout estrous cycle. J Endocrinol Invest. 1995; 18(11): 853-6.

10. Stats, A. Federal Interagency Forum on Aging Related Statistics. Retrieved online April. 2006;22:2009.

11. Van Dam, E.W., Dekker, J.M., Lentjes, E.G., Romijn, F.P., Smulders, Y.M., Post, W.J. et al. Steroids in Adult Men With Type 1 Diabetes A tendency to hypogonadism. Diabetes Care. 2003;26(6):1812-8.

12. Mattson, M.P., Maudsley, S., Martin, B. BDNF and 5-HT: a dynamic duo in age-related neuronal plasticity and neurodegenerative disorders. Trends Neurosci. 2004;27(10):589-94.

13. Tyler, W.J., Alonso, M., Bramham, C.R., PozzoMiller, L.D. From acquisition to consolidation: on the role of brain-derived neurotrophic factor signaling in hippocampal-dependent learning. Learn Mem. 2002; 9(5): 224-37.

14. Conner, J.M., Lauterborn, J.C., Yan, Q., Gall, C.M., Varon, S. Distribution of brain-derived neurotrophic factor (BDNF) protein and mRNA in the normal adult rat CNS: evidence for anterograde axonal transport. J Neurosci 1997; 17(7):2295-313.

15. Kang, H., Schuman, E.M. Long-lasting neurotrophin-induced enhancement of synaptic transmission in the adult hippocampus. Science. 1995; 267(5204):1658-62.

16. Fleischer, A., Ghadiri, A., Dessauge, F., Duhamel, M., Rebollo, M.P., Alvarez-Franco, F. et al. Modulating apoptosis as a target for effective therapy. Mol Immunol. 2006;43(8):1065-79.

17. Tsai, S-J. Brain-derived neurotrophic factor: a bridge between major depression and Alzheimer's disease? Med Hypotheses. 2003; 61(1): 110-3.

18. Navaratna, D., Guo, S-z., Hayakawa, K., Wang, X., Gerhardinger, C., Lo, E.H. Decreased Cerebrovascular Brain-Derived Neurotrophic Factor-Mediated Neuroprotection in the Diabetic Brain. Diabetes. 2011;60(6):1789-96.

19. Oghbaei, H., Asl, N.A., Sheikhzadeh, F., Alipour, M.R., Khamaneh, A.M. The Effect of Regular Moderate Exercise on miRNA-192 Expression Changes in Kidney of Streptozotocin-Induced Diabetic Male Rats. 
Adv Pharm Bull. 2015.

20. Li, M., Masugi-Tokita, M., Takanami, K., Yamada, S., Kawata, M. Testosterone has sublayer-specific effects on dendritic spine maturation mediated by BDNF and PSD-95 in pyramidal neurons in the hippocampus CA1 area. Brain research. 2012;1484:76-84.

21. Pourrabi, S., Nayebi, A.M., Hossini, S. Role of the Androgen Receptors in Memory Impairment in Mature Male Rats. 1(2):25-32.

22. Besheer, J., Jensen, H.C., Bevins, R.A. Dopamine antagonism in a novel-object recognition and a novel-object place conditioning preparation with rats. Behav Brain Res. 1999; 103(1): 35-44.

23. Buckmaster, C.A., Eichenbaum, H., Amaral, D.G., Suzuki, W.A., Rapp, P.R. Entorhinal cortex lesions disrupt the relational organization of memory in monkeys. J Neurosci. 2004; 24(44): 9811-25.

24. Maisonpierre, P.C., Le Beau, M.M., Espinosa, R., Ip, N.Y., Belluscio, L., Suzanne, M., et al. Human and rat brain-derived neurotrophic factor and neurotrophin-3: gene structures, distributions, and chromosomal localizations. Genomics. 1991; 10(3): 558-68.

25. de Lima, M.N.M., Laranja, D.C., Bromberg, E., Roesler, R., Schröder, N. Pre-or post-training administration of the NMDA receptor blocker MK-801 impairs object recognition memory in rats. Behav Brain Res. 2005; 156(1): 139-43.

26. Karasawa, J-i., Hashimoto, K., Chaki, S. DSerine and a glycine transporter inhibitor improve MK-801-induced cognitive deficits in a novel object recognition test in rats. Behav Brain Res. 2008; 186(1): 78-83.

27. Brands, A.M., Biessels, G.J., De Haan, E.H., Kappelle, L.J., Kessels, R.P. The effects of type 1 diabetes on cognitive performance A metaanalysis. Diabetes Care. 2005; 28(3): 726-35.

28. Akbarzadeh, A., Norouzian, D., Mehrabi, M., Jamshidi, S., Farhangi, A., Verdi, A.A., et al. Induction of diabetes by streptozotocin in rats. Indian J Clin Biochem. 2007;22(2):60-4.

29. Szkudelski, T. The mechanism of alloxan and streptozotocin action in B cells of the rat pancreas. Physiol Res. 2001;50(6):537-46.

30. Grossmann, M., Thomas, M.C., Panagiotopoulos, S., Sharpe, K., MacIsaac, R.J., Clarke, S., et al. Low testosterone levels are common and associated with insulin resistance in men with diabetes. J Clin Endocrinol Metab. 2013.

31. Naghdi, N., Majlessi, N., Bozorgmehr, T. The effect of intrahippocampal injection of testosterone enanthate (an androgen receptor agonist) and anisomycin (protein synthesis inhibitor) on spatial learning and memory in adult, male rats. Behav Brain Res. 2005;156(2):263-8.

32. Emamian, S., Naghdi, N., Sepehri, H., Jahanshahi, M., Sadeghi, Y., Choopani, S. Learning impairment caused by intra-CA1 microinjection of testosterone increases the number of astrocytes. Behav Brain Res. 2010;208(1):307.

33. Harooni, H.E., Naghdi, N., Rohani, A.H. Intra hippocampal injection of testosterone impaired acquisition, consolidation and retrieval of inhibitory avoidance learning and memory in adult male rats. Behav Brain Res. 2008; 188(1): 71-7.

34. Alexander, G.M., Packard, M.G., Hines, M. Testosterone has rewarding affective properties in male rats: implications for the biological basis of sexual motivation. Behav Neurosci. 1994; 108(2): 424.

35. Sandstrom, N.J., Kim, J.H., Wasserman, M.A. Testosterone modulates performance on a spatial working memory task in male rats. Horm Behav. 2006; 50(1): 18-26.

36. Hawley, W.R., Grissom, E.M., Martin, R.C., Halmos, M.B., Bart, C.L., Dohanich, G.P. Testosterone modulates spatial recognition memory in male rats. Horm Behav. 2013; 63(4): 559-65.

37. Daniel, J.M., Winsauer, P.J., Moerschbaecher, J.M. Castration in rats impairs performance during acquisition of a working memory task and exacerbates deficits in working memory produced by scopolamine and mecamylamine. Psychopharmacology. 2003; 170(3): 294-300.

38. Aubele, T., Kaufman, R., Montalmant, F., Kritzer, M. Effects of gonadectomy and hormone replacement on a spontaneous novel object recognition task in adult male rats. Horm Behav. 2008; 54(2): 244-52.

39. Frye, C.A., Lacey, E.H. Posttraining androgens' enhancement of cognitive performance is temporally distinct from androgens' increases in affective behavior. Cogn Affect Behav Neurosci. 2001; 1(2): 172-82.

40. Pourrabi, S., Nayebi, A.M., Hossini, S. Role of the Androgen Receptors in Memory Impairment in Mature Male Rats.

41. Spritzer, M.D., Galea, L.A. Testosterone and dihydrotestosterone, but not estradiol, enhance survival of new hippocampal neurons in adult male rats. Dev Neurobiol. 2007;67(10):1321-33.

42. Fowler, C.D., Freeman, M.E., Wang, Z. Newly proliferated cells in the adult male amygdala are affected by gonadal steroid hormones. $J$ Neurobiol. 2003;57(3):257-69. 
43. Rasika, S., Nottebohm, F., Alvarez-Buylla, A. Testosterone increases the recruitment and/or survival of new high vocal center neurons in adult female canaries. Proc Natl Acad Sci. 1994; 91(17):7854-8.

44. Estrada, M., Varshney, A., Ehrlich, B.E. Elevated testosterone induces apoptosis in neuronal cells. J Biol Chem. 2006;281(35):25492-501.

45. Lee, J., Duan, W., Mattson, M.P. Evidence that brain derived neurotrophic factor is required for basal neurogenesis and mediates, in part, the enhancement of neurogenesis by dietary restriction in the hippocampus of adult mice. $J$ Neurochem. 2002;82(6):1367-75.

46. Cameron, H., Woolley, C., McEwen, B., Gould, E. Differentiation of newly born neurons and glia in the dentate gyrus of the adult rat. Neuroscience. 1993;56(2):337-44.

47. Kalaria, R.N. Neurodegenerative disease: diabetes, microvascular pathology and Alzheimer disease. Nat Rev Neurol. 2009; 5(6): 305-6.

48. Phillips, H.S., Hains, J.M., Laramee, G.R., Rosenthal, A., Winslow, J.W. Widespread expression of BDNF but not NT3 by target areas of basal forebrain cholinergic neurons.
Science. 1990; 250(4978): 290-4.

49. Villani, G.R., Gargiulo, N., Faraonio, R., Castaldo, S., Gonzalez, y. Reyero, E., Di, Natale, P. Cytokines, neurotrophins, and oxidative stress in brain disease from mucopolysaccharidosis IIIB. J Neurosci Res. 2007; 85(3): 612-22.

50. Larsson, E., Nanobashvili, A., Kokaia, Z., Lindvall, O. Evidence for neuroprotective effects of endogenous brain-derived neurotrophic factor after global forebrain ischemia in rats. $J$ Cereb Blood Flow Metab. 1999;19(11):1220-8.

51. Nitta, A., Murai, R., Suzuki, N., Ito, H., Nomoto, H., Katoh, G., et al. Diabetic neuropathies in brain are induced by deficiency of BDNF. Neurotoxicol Teratol. 2002;24(5):695-701.

52. Rasika, S., Alvarez-Buylla, A., Nottebohm, F. BDNF mediates the effects of testosterone on the survival of new neurons in an adult brain. Neuron. 1999;22(1):53-62.

53. Ottem, E.N., Beck, L.A., Jordan, C.L., Breedlove, S.M. Androgen-dependent regulation of brain-derived neurotrophic factor and tyrosine kinase $\mathrm{B}$ in the sexually dimorphic spinal nucleus of the bulbocavernosus. Endocrinology. 2007;148(8):3655-65. 